International Journal of Modern Physics A,

UR-1619

(C) World Scientific Publishing Company

$\mathrm{ER} / 40685 / 957$

December 2000

\title{
TOP PRODUCTION AND DECAY AT LINEAR COLLIDERS: QCD CORRECTIONS*
}

\author{
COSMIN MACESANU and LYNNE H. ORR \\ Department of Physics and Astronomy, University of Rochester \\ Rochester NY 14627-0171, USA
}

\begin{abstract}
We present the results of an exact calculation of gluon radiation in top production and decay at high energy electron-positron colliders. We include all spin correlations and interferences, the bottom quark mass, and finite top width effects in the matrix element calculation. We study properties of the radiated gluons and implications for top mass measurement. We also discuss virtual corrections to the process.
\end{abstract}

\section{Introduction}

Lepton colliders provide a clean environment for top quark studies. In constrast to hadron colliders, kinematics and QCD backgrounds are well under control at lepton colliders. This allows for measurements of top quark properties such as mass, width, and electromagnetic couplings. These measurements require precise predictions, beyond leading order in perturbation theory. Furthermore, because the top quark is a heavy, unstable particle, higher-order corrections to both the top production and decay processes must be considered together. This is becoming the standard approach as we enter the $\mathrm{TeV}$ regime and is already familiar from dealing with $Z$ and $W W$ production at LEP.

Here we consider QCD corrections to $t \bar{t}$ production and decay at linear colliders at energies above the top pair thresholdl; see also 2 . For most of the talk we consider real gluon corrections, and comment briefly on virtual corrections at the end.

\section{Real Gluon Radiation}

Radiated gluons appear as hadronic jets in particle detectors, and these jets are often indistinguishable from the jets coming from top quark decays (indeed, the gluons may be part of the decays themselves). As a result, identification of top quark events and measurement of the top mass by momentum reconstruction can be complicated by the presence of gluon radiation.

At lepton colliders there can be no gluon radiation from the initial state, but gluons can be radiated from the produced quarks in either the top production or decay stage. We have calculated the cross section for the process

$$
e^{+} e^{-} \rightarrow \gamma^{*}, Z^{*} \rightarrow t \bar{t}(g) \rightarrow b W^{+} \bar{b} W^{-} g .
$$

using the exact matrix elements including top width effects, spin correlations between top production and decay, all masses, and all interferencest.

*Presented at the 2000 Meeting of the Division of Particles and Fields of the APS, Columbus, OH, August 9-12, 2000. 


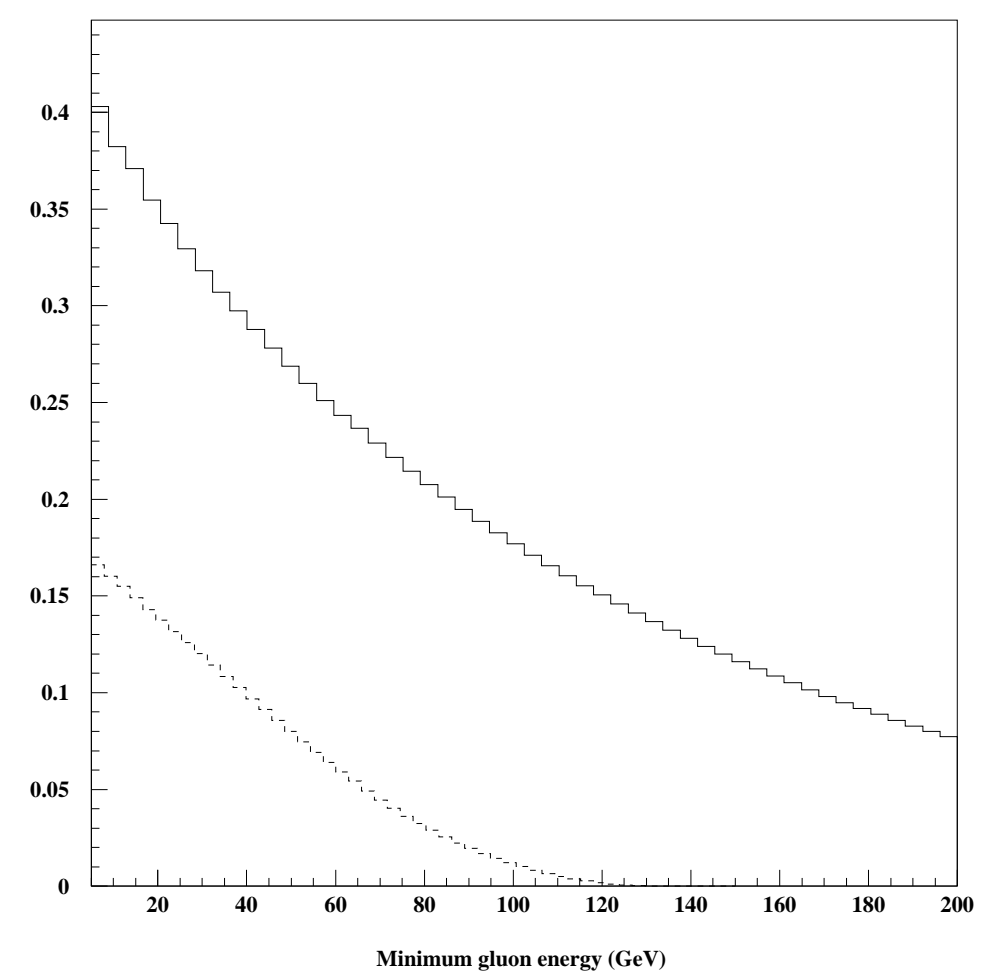

Figure 1: The fraction of gluon emissions radiated in the production stage, as a function of minimum gluon energy, for center-of-mass energy $1 \mathrm{TeV}$ (solid line) and $500 \mathrm{GeV}$ (dashed line).

The distinction between production- and decay-stage emissions is based on whether the top quark is closer to being on shell before or after the top quark is radiated. In the calculation the separation is straightforward with a little algebra; we can then identify the individual contributions from the production and decay stages as well as their interferences (which are small for the most part).

We show in Figure 1 the resulting fraction of the cross section contributed by production-stage radiation as a function of the minimum gluon energy. We see that gluons emitted in the production stage account for less than half of the radiative cross section in both cases. Phase space accounts both for the increase in the fraction with center of mass energy and the decrease with increasing gluon energy.

Results for mass reconstruction are shown in Figure 2, with and without the extra gluon included. We see clear peaks where the correct combination is obtained, with a low tail when decay-stage gluons are omitted, and a high tail where production-stage gluons are included. An invariant mass cut can be used to improve the resolution and angular cuts (between the gluon and the $b$ quarks) can do even better. Our results are at the parton level however, so hadronization and detector effects must ultimately be taken into account.

The interference between gluons radiated in the production and decay stages, 


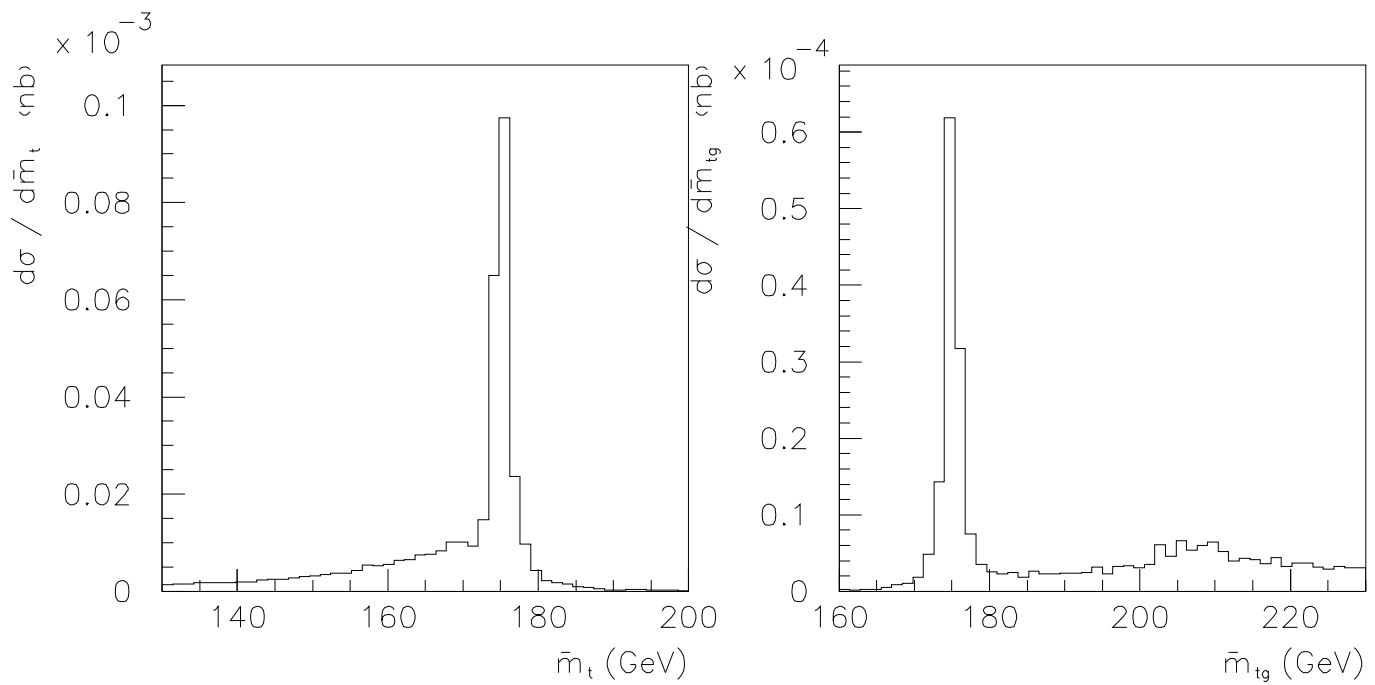

Figure 2: The top invariant mass spectrum without (left) and with (right) the gluon momentum included, for center-of-mass energy $600 \mathrm{GeV}$.

though small, can be interesting because it is sensitive to the total width of the top quark. In particular, this interference is largest when the gluon energy is comparable to the top width, and given suitable cuts, can be destructive. See 1 for details.

\section{Virtual Corrections}

A complete next-to-leading order calculation requires virtual corrections, and these are currently in progress 3 . Individual corrections to production and decayl (socalled "factorizable" corrections) have been known for some time. But the interferencetype or "nonfactorizable" corrections, where for example a gluon connects the top quark and the bottom antiquark, have not. Many of the theoretical issues are similar to those that arise in the calculation of corrections to $W W$ production at LEPE. As in the $W W$ case, the calculation is being performed in the Double-Pole Approximation, in which only contributions from doubly-resonant diagrams are kept. The complete calculation will be aviailable soon.

\section{Acknowledgments}

Work supported in part by the U.S. Department of Energy and the U.S. National Science Foundation, under grants DE-FG02-91ER40685 and PHY-9600155.

1. C. Macesanu and L.H. Orr, hep-ph/0012177.

2. C.R. Schmidt, Phys. Rev. D54 3250 (1996).

3. C. Macesanu, hep-ph/0012173 and in preparation.

4. J. Jersak, E. Laerman, and P. Zerwas, Phys. Rev. D25 1218 (1982).

5. M. Jezabek and J. Kuhn, Phys. Lett. B329 317 (1994).

6. D. Wackeroth, talk at this conference (Denner et al., hep-ph/0011119 and references therein). 